> Les dernières études menées sur la leucémie myéloïde chronique (LMC) ont permis de mettre en évidence le rôle clé des cellules NK (natural killer) dans cette maladie avec trois enseignements majeurs: (1) au diagnostic, les cellules NK sont anormales dans leurs propriétés phénotypiques et fonctionnelles ; (2) un traitement standard par inhibiteur de tyrosine kinase (ITK) est associé à la correction de certaines anomalies, en particulier celles des lymphocytes NK; (3) le taux du soustype mature NK CD56 dim pourrait être un facteur du maintien d'une survie sans rechute après une stratégie d'arrêt de traitement par ITK. Ces données renforcent le rôle des lymphocytes NK dans le contrôle immunologique de la LMC. <

La leucémie myéloïde chronique (LMC) est une hémopathie maligne clonale initiée par la fusion des gènes $B C R$ (breakpoint cluster region) et $A B L I$ (Abelson), conséquence de la translocation réciproque entre le chromosome 9 et le chromosome 22 - $t(9 ; 22)(q 34 ; q 11)$ - dans une cellule souche hématopoïétique. L'oncogène chimérique $B C R-A B L 1$ qui en résulte code une protéine de fusion (BCR-ABL) dont l'activité tyrosine kinase est dérégulée [1]. Depuis le début des années 2000, les patients atteints de LMC bénéficient d'une thérapie ciblée fondée sur l'utilisation d'inhibiteurs de tyrosine kinase (ITK). L'inhibiteur de première génération, l'imatinib, a constitué une véritable révolution dans la prise en charge thérapeutique et le pronostic de la LMC, de telle sorte que l'espérance de vie des patients diagnostiqués en phase chronique de la maladie et répondeurs aux traitements se rapproche de celle de la population générale [2, 3]. D’autres ITK (dasatinib, nilotinib, bosutinib, ponatinib) ont été développés pour surmonter les résistances à ce premier inhibiteur [4].

Ces dernières années, divers travaux ont permis de montrer que les ITK exercent leur action à deux niveaux: par inhibition directe des kinases (BCR-ABL, Src, c-kit, Tec) [5] au niveau des cellules leucémiques, mais également

Vignette (Photo ( $)$ Inserm - Céline Richard/Plateforme d'imagerie de I'Institut Cochin).

\section{Lymphocytes NK : un rôle majeur dans le contrôle immunologique de la leucémie myéloïde chronique}

\author{
Antoine Toubert ${ }^{1-3}$, Ali Turhan ${ }^{4,5}$, \\ Agnès Guerci-Bresler ${ }^{6,7}$, Nicolas Dulphy ${ }^{1-3}$, \\ Delphine Réa ${ }^{1,7,8}$
}

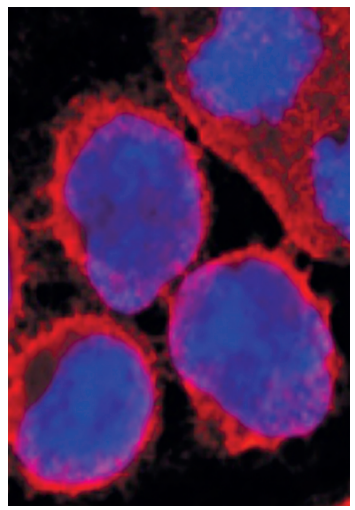

par un effet immunomodulateur concomitant [1]. Un certain nombre d'anomalies phénotypiques et fonctionnelles des cellules du système immunitaire ont été mises en évidence chez les patients atteints de LMC au diagnostic, perturbations qui s'aggravent avec la progression de la maladie [1]. Parmi elles, on observe un déséquilibre fonctionnel et quantitatif des cellules natural killer (NK), première ligne de défense antitumorale. Cette population de cellules immunitaires semble en effet jouer un rôle central dans la réponse anti-tumorale, laissant entrevoir le développement de nouvelles approches thérapeutiques d'immunothérapie $[1,6]$. Cette revue a pour objectif de synthétiser les connaissances actuelles dans ce domaine.

${ }^{1}$ Inserm UMR 1160, Hôpital Saint-Louis, 1, avenue Claude Vellefaux, 75010 Paris, France. 'Laboratoire d'immunologie et d'histocompatibilité, Hôpital Saint-Louis, 1, avenue Claude Vellefaux, 75010 Paris, France. ${ }^{3}$ Institut universitaire d'hématologie, Université Paris Diderot, Sorbonne Paris Cité, 1, avenue Claude Vellefaux, 75010 Paris, France.

${ }^{4}$ Institut fédératif d'hématologie Paris Sud et service

d'hématologie, Hôpitaux

Universitaires Paris Sud, Kremlin Bicêtre et Paul Brousse, 94800 Villejuif France. ${ }^{5}$ Inserm U935, Campus CNRS, 94800 Villejuif, France. 'Service d'hématologie, CHRU Brabois, 54500 Vandœuvre-lèsNancy, France.

${ }^{7}$ France intergroupe des leucémies myéloïdes chroniques (Fi-LMC), Institut Bergonié, 33000 Bordeaux, France. ${ }^{8}$ Service d'hématologie adulte, Hôpital Saint-Louis, 1, avenue Claude Vellefaux, 75010 Paris, France.

antoine.toubert@univ-parisdiderot.fr

\section{La cellule NK : un système de} détection hautement sophistiqué dans la défense immunitaire anti-tumorale

Découvertes depuis plus de 40 ans, les cellules natural killer (NK) font l'objet d'une attention croissante en raison de leur potentiel rôle 
immunothérapeutique. Elles font partie du système de défense immunitaire anti-tumorale, et sont, à présent, incluses dans la famille des cellules lymphoïdes innées (ILC) [7].

Reconnues phénotypiquement comme $\mathrm{CD}^{-} \mathrm{CD}_{5} 6^{+} \mathrm{CD} 16^{+/-}$, les cellules NK humaines sont divisées en deux sous-groupes, les CD56 $6^{\text {bright }}$ et les CD56 $6^{\mathrm{dim}}$. Les NK CD56 $6^{\mathrm{dim}}$ sont les plus nombreuses dans le sang. Elles sont les plus matures et sont caractérisées par une activité cytotoxique plus efficace [7].

D'un point de vue fonctionnel, les cellules NK distinguent les cellules saines des cellules anormales par un système hautement sophistiqué résultant du rapport entre signaux activateurs et signaux inhibiteurs perçus via une multitude de récepteurs situés à leur surface [7-9] (Figure 1). Grâce à ces récepteurs, elles peuvent détecter les modifications survenant dans les cellules transformées, notamment une diminution d'expression de molécules du complexe d'histocompatibilité de classe I (CMH-I) et/ou l'expression accrue des molécules induites par le stress tumoral telles que MICA et MICB (MHC class I polypeptide-related sequence $A, B$ ) [10], ligands du récepteur activateur NKG2D (natural killer group 2D) [7]. Les mécanismes majeurs impliqués sont les suivants: (1) action cytotoxique directe sur les cellules tumorales par libération du contenu enzymatique de leurs granules cytoplasmiques (perforine, granzyme) et induction d'apoptose de la cible, production de molécules effectrices, et ADCC (cytotoxicité cellulaire dépendante d'anticorps); (2) action régulatrice indirecte par interaction avec diverses populations cellulaires immunitaires via la production de cytokines (IFN $\gamma$ [interféron gamma], TNF $\alpha$ [tumor necrosis factor alpha]), de chimiokines ou de facteurs de croissance, stimulation de la présentation des antigènes par les cellules dendritiques, activation des lymphocytes $\operatorname{TCD}^{+}$en CTL (lymphocytes T cytotoxiques), induction d'une différenciation des lymphocytes $T$ CD4 $4^{+}$en lymphocytes de type Thl, régulation de la production d'anticorps anti-tumoraux par les lymphocytes $B$ (Figure 2) [11].

L'intérêt des cellules NK dans l'immunomodulation anti-tumorale trouve sa source dans différents travaux. Au cours du développement de tumeurs solides, la fonction des cellules NK (et des cellules T cytotoxiques) est profondément altérée dans le cadre de l'«immunoediting » tumoral ${ }^{1}$. Une cytotoxicité défectueuse et une modulation d'expression des récepteurs de surface ont également été rapportées dans les cellules NK chez les patients atteints de cancer avant traitement. Enfin, une faible fonction NK prédit un risque plus élevé de développer un cancer $[7,8]$. En outre, contrairement aux lymphocytes $\mathrm{TCD}^{+}$, qui requièrent un mécanisme long et complexe de préactivation pour acquérir un statut d'acteur cytotoxique efficace, les cellules NK sont «prêtes à tuer » à l'issue de leur processus d'éducation, ce qui leur confère un avantage puissant pour être utilisées dans des approches immunothérapeutiques [7].

${ }^{1}$ L'« immunoediting 》 définit les changements de l'immunogénicité d’une tumeur induits par la réponse immunitaire anti-tumorale.

\section{La LMC : un déficit quantitatif et fonctionnel des NK associé à l'évolution de la maladie}

Comme dans les tumeurs solides, on observe un dysfonctionnement ainsi qu'une diminution du nombre de cellules NK chez les patients atteints de LMC en phase chronique, au moment du diagnostic $[1,12]$. Dans un modèle murin de LMC, une diminution de la dégranulation des cellules NK est également observée [13]. Chez les patients, les travaux s'intéressant aux mécanismes du dysfonctionnement des cellules NK montrent une perte d'expression par ces cellules, des récepteurs activateurs NKG2D, CD94-NKG2C, NKp30, NKp46 ou KIR (killer cell immunoglobulin-like receptor) $[1,14,15]$. En outre, les déficits quantitatifs et fonctionnels décrits s'aggravent avec la progression de la maladie vers les phases avancées et blastiques [16]. L'ensemble de ces données confirme donc un rôle central des cellules NK dans la physiopathologie de la LMC.

\section{ITK : effets sur la mobilisation et la fonction NK et T dans la LMC}

Les ITK ont révolutionné la prise en charge des patients atteints de LMC et ont remplacé l'IFN $\alpha$ comme traitement de première ligne [1]. Rappelons que l'efficacité clinique des ITK est évaluée en mesurant la réduction du nombre de cellules leucémiques circulantes par la détermination par PCR (polymerase chain reaction) quantitative de la quantité de transcrits $B C R-A B L 1$ [17]. L'obtention d'une réponse optimale après l'initiation du traitement constitue l'objectif majeur de la stratégie thérapeutique afin d'augmenter la survie sans progression. La réponse optimale correspond à une réponse moléculaire majeure (RMM) définie par un niveau de transcrits $B C R-A B L 1$ $\leqslant 0,1 \%$. Un objectif plus récent dans le traitement de la LMC est d'obtenir une réponse moléculaire profonde (RMP) stable et durable pendant au moins 2 ans, prérequis pour une rémission sans traitement (RST), qui est observée chez environ $50 \%$ des patients qui arrêtent leur traitement dans ces conditions [1]. La raison principale de la survenue des rechutes, malgré l'absence de transcrit $B C R-A B L 1$ détectable chez ces patients, est la persistance de cellules souches leucémiques au niveau médullaire [18], notamment en raison de leur résistance aux ITK utilisés en clinique $[19,20]$. En effet, l'arrêt du traitement par les ITK en situation de RMP conduit à des rechutes rapides, dans plus de la moitié des cas, dans tous les essais cliniques [21]. En revanche, l'absence de rechute chez certains de ces patients a été attribuée, en partie, à un contrôle immunologique de la maladie résiduelle [1]. 


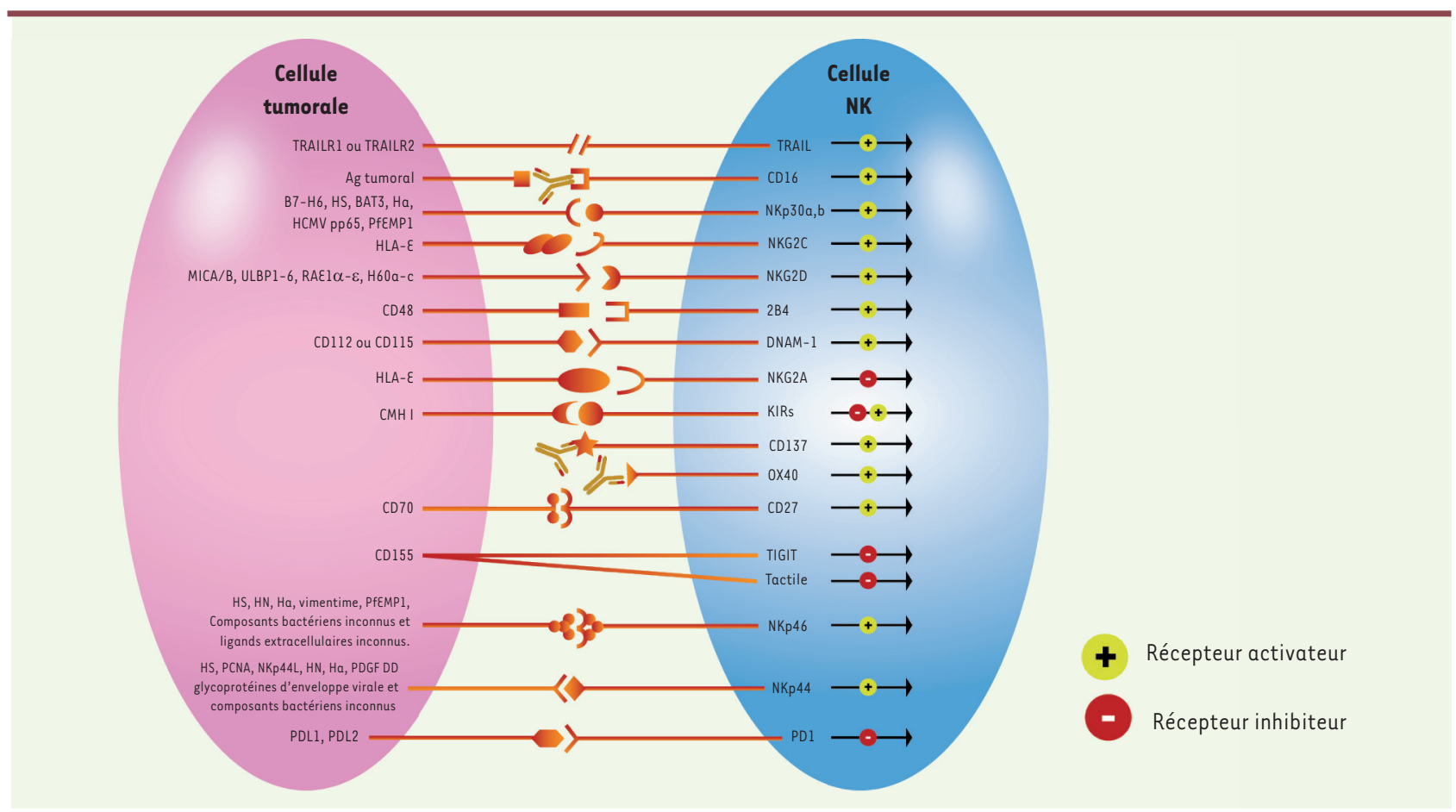

Figure 1. Les récepteurs des cellules NK et leurs ligands (adaptée d'une illustration originalement publiée par Chester et al. [9] et de données issues de Morvan et al. [7]). TRAIL : TNF-related apoptosis-inducing ligand ; TRAILR: TRAIL receptor; IgG : immunoglobuline G ; HS : héparine sulfate; Ha : hémagglutinine ; HCMV : human cytomegalovirus; PfEMPI : Plasmodium falciparum erythrocyte membrane protein 1 ; HLA- $\varepsilon$ : HLA class I histocompatibility antigen, alpha chain $\varepsilon$; MICA/B : MHC class I polypeptide-related sequence A/B; ULBP : UL16 binding protein 1 ; RAE $\alpha-\varepsilon$ : retinoic acid early inducible gene-1 $\alpha-\varepsilon ; C M H-I$ : complexe majeur d'histocompatibilité (CMH) de classe I; HN : hémagglutinine neuraminidase ; NK : natural killer; KIR : killer-cell immunoglobulin-like receptor; PCNA : proliferating cell nuclear antigen; PD-1 : programmed cell death-1; PD-L1/2 : programmed cell death-ligand 1/2; PVR : poliovirus receptor; Tactile : T cell activation, increased late expression ; TIGIT : T cell immunoreceptor with Ig and ITIM domains; DNAM-1 : DNAX accessory molecule-1 ; NKG2 : natural killer group 2.

In vitro, alors que l'imatinib ne montre pas d'effet sur les cellules NK, le dasatinib inhibe leurs capacités cytotoxiques et de production de cytokines [22]. Le nilotinib induit également une diminution de la production des cytokines par les cellules NK, mais n'a pas d'effet sur la cytotoxicité [22]. L'imatinib et le dasatinib inhibent aussi la prolifération des lymphocytes $T$, les effets du dasatinib étant plus profonds, probablement en raison d'une action inhibitrice supplémentaire sur les kinases Src [23]. In vivo, Rohon et al. ont montré que l'imatinib induit une normalisation du profil immunologique des patients atteints de LMC, celui-ci devenant similaire à celui des sujets contrôles, alors qu'au diagnostic, une faible proportion de lymphocytes B et de cellules dendritiques et une augmentation des lymphocytes NK/T étaient observées dans la moelle osseuse [5]. Dans deux autres études [14, 24], une réponse CTL a été révélée chez la majorité des patients en rémission sous imatinib. Selon Hughes et al., cette restauration de la réponse $T$ cytotoxique spécifique d'antigènes leucémiques pourrait être liée à la levée de voies immunosuppressives impliquant le récepteur PD-1 (programmed cell death-1) [14]. Selon cette étude, les patients en RMM et en RMP présentent des cellules NK dont le phénotype est plus mature et plus cytolytique ( $\left.C D 57^{+} \mathrm{CD} 62 \mathrm{~L}^{-}\right)$après traitement par ITK (imatinib, dasatinib, nilotinib), avec une expression restaurée des récepteurs KIR2D (killer cell immunoglobulin like receptor, two Ig domains) -L2/L3 (and long cytoplasmic tail 2, 3) et -S2 (and short cytoplasmic tail 2), rendant les cellules NK plus fonctionnelles [25], ce qui est cohérent avec la restauration d'un répertoire NK activateur et inhibiteur [14].

De nombreux travaux convergents mettent en évidence l'induction par le dasatinib d'une hyperlymphocytose (4 à $20 \times 10^{9}$ lymphocytes/I) fréquente et réversible, caractérisée par une expansion de clones de lymphocytes dits LGL (large granular lymphocytes) mono ou oligoclonaux (lymphocytes $\mathrm{T} \mathrm{CD} 3^{+} \mathrm{CD} 8^{+}$ou cellules NK $\left.\mathrm{CD}^{-}{ }^{-} \mathrm{CD} 16^{+} \mathrm{CD}^{\circ} 6^{+}\right)$[26].

Au-delà de ces divers effets, les ITK modulent aussi les mécanismes immunosuppresseurs des cellules tumorales. Le dasatinib et l'imatinib peuvent induire une diminution du nombre de cellules myéloïdes suppressives (MDSC, myeloid-derived suppressor cells) associée à l'expression de la molécule inhibitrice arginase-1 [27]. Rappelons que les MDSC favorisent le recrutement et l'expansion d'autres cellules suppressives (les 


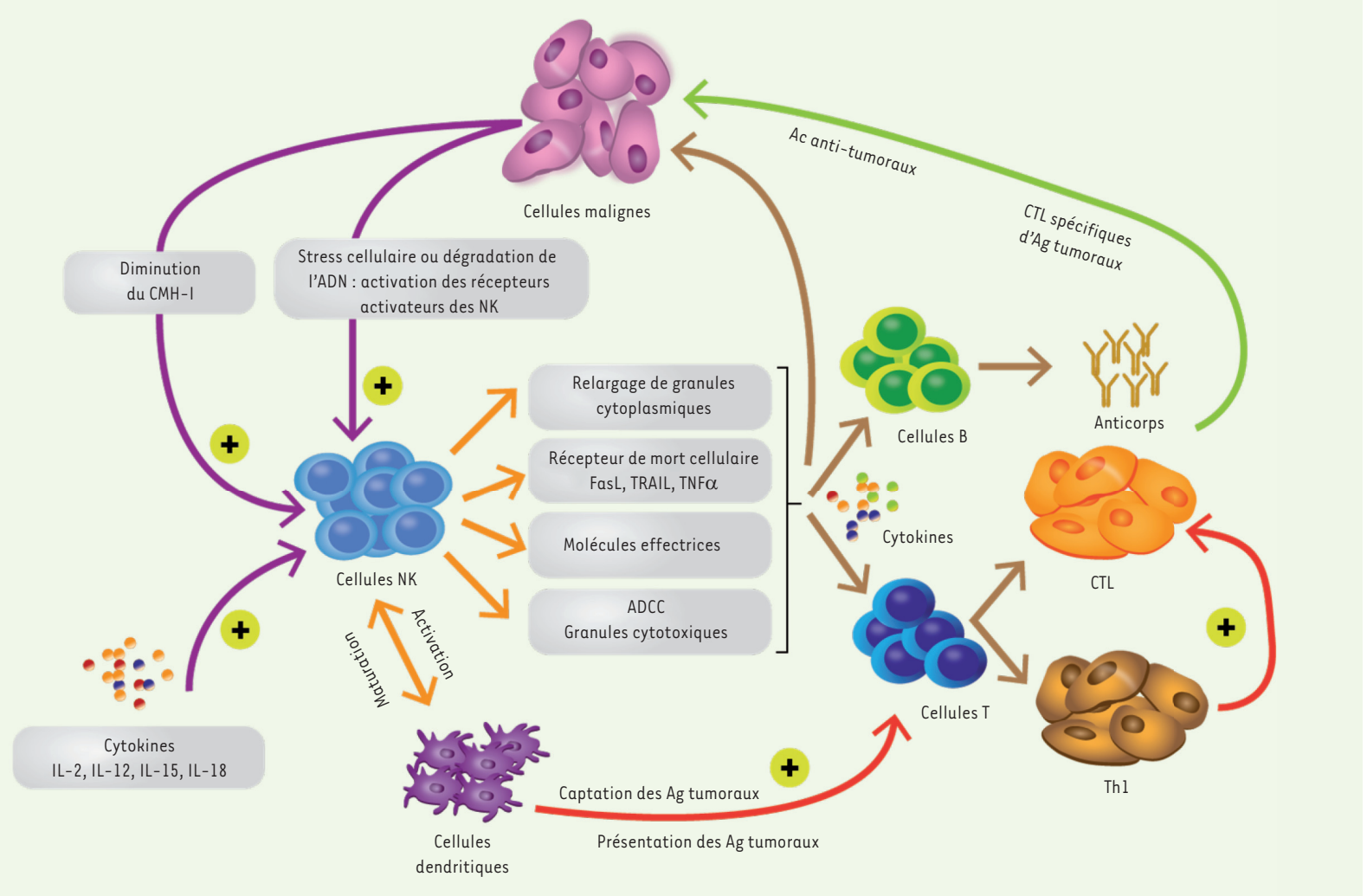

Figure 2. Immunosurveillance anti-tumorale par les cellules NK (adaptée de Cheng et al. [11]). IL: interleukine; CMH-I: complexe majeur d'histocompatibilité de classe I; CTL : cytotoxic T lymphocyte ; Ac : anticorps ; Ag : antigène ; NK : natural killer ; ADCC : antibody-dependent cellmediated cytotoxicity; TRAIL : TNF-related apoptosis-inducing ligand; TNF $\alpha$ : tumor-necrosis-factor $\alpha$; FasL : Fas ligand.

cellules T régulatrices ou Treg), conduisant à la détérioration des cellules NK et à l'inhibition de l'activation et de la prolifération des lymphocytes T [1]. Le dasatinib a la capacité de réduire le nombre de Treg dans la moelle osseuse et le sang, en inversant l'équilibre entre suppression et stimulation immunitaire [28]. La réduction du nombre de Treg serait, par ailleurs, marquée chez les patients traités par dasatinib et qui développent une lymphocytose à $\operatorname{LGL}[29,30]$.

\section{Hyperlymphocytose : facteur prédictif de la réponse au traitement dans la LMC?}

La lymphoprolifération de type LGL induite par le dasatinib est une expansion de clones LGL (NK et T CD8 $8^{+}$) pré-existants au moment du diagnostic de LMC (on les retrouve chez $80 \%$ des patients) [30]. Alors que chez les patients traités par dasatinib, on note une expansion de ces clones, on n'observe aucun changement chez les patients traités par imatinib ou nilotinib [30]. Cette mobilisation peut être transitoire ou persistante. Mustjoki et al. ont montré, chez des patients atteints de LMC et de LAM (leucémie aiguë myéloblastique), qu'elle survenait $\mathrm{l}$ à $2 \mathrm{~h}$ après traitement par dasatinib, avec une prédominance des cellules NK $\left(\mathrm{CD}^{-} \mathrm{CD} 16^{+} \mathrm{CD} 56^{+}\right)$et des lymphocytes B $\left(\mathrm{CD} 19^{+}\right)[29]$. Pour d'autres auteurs, l'expansion se révèle entre 1 mois et 18 mois après le début du traitement, la variation des données étant liée à la méthodologie choisie [30,31]. Chez la plupart des patients, cette expansion de clones LGL perdure tout au long du traitement par dasatinib, bien que des fluctuations marquées soient observées quant au nombre absolu de lymphocytes. Elle ne persiste pas après arrêt du traitement, le taux de clones LGL revenant quasiment aux niveaux observés avant l'instauration du traitement [30].

L'expansion de clones LGL est associée à une augmentation de l'activité cytotoxique des cellules NK $[32,33]$ et à une diminution des Treg dans le sang [30]. Elle semble également corrélée à une réponse moléculaire plus favorable [30]. Plusieurs études montrent en effet une augmentation significative de RMM et de RMP chez les patients présentant une hyperlymphocytose, comparativement à ceux qui en sont exempts [30]. Iryama et al. montrent, quant à eux, que le nombre de cellules NK et de cellules cytotoxiques CTL est significativement plus élevé chez les patients en RMP à 18 mois par rapport aux patients chez qui cette réponse n'est pas obtenue. $\varepsilon$ n revanche, cette étude montre que le nombre de cellules 
Treg est significativement plus faible à 12 mois et 15 mois chez les patients en RMP [31].

Comment interpréter cette immunomodulation spécifique du dasatinib ? Le dasatinib, au-delà de l'inhibition de la tyrosine kinase BCR$A B L$, inhibe également les kinases Src, Fyn ou Lck (lymphocyte-specific protein tyrosine kinase). L'imatinib et le nilotinib, qui n'induisent pas ce phénomène de lymphoprolifération, n'ont pas la capacité d'inhiber les kinases de la famille Src. Selon Qiu et al., la modulation des kinases de la famille Src par le dasatinib pourrait déréguler l'activation/ prolifération des cellules NK ou NK/T et, ainsi, stimuler les fonctions anti-leucémiques des LGL [30]. II reste toutefois à confirmer cette hypothèse et à préciser si l'effet du dasatinib sur l'activation/prolifération des cellules NK ou NK/T implique les kinases Src ou une autre voie de signalisation.

\section{Arrêt de traitement dans la LMC : le statut NK a-t-il une valeur prédictive de rémission?}

L'imatinib permet d'améliorer considérablement la survie des patients atteints de LMC. Les ITK de seconde et troisième génération permettent d'obtenir plus rapidement une RMP [1]. Néanmoins, il est encore recommandé aujourd'hui d'administrer le traitement au long cours, voire toute la vie, en raison de l'incapacité de l'imatinib, et des autres ITK, à éliminer les cellules souches leucémiques quiescentes au niveau médullaire [3], ce qui entraîne des conséquences pour les patients, en termes de tolérance et de qualité de vie, et pour la société, en termes économiques. C'est pourquoi, ces dernières années, ont été testées des stratégies d'arrêt de traitement chez les patients en RMP durable. Plusieurs études, dont STIM (Stop Imatinib) et TWISTER, ont démontré que $40 \%$ des patients atteints de LMC en RMP durable pouvaient maintenir cette réponse sans traitement après arrêt de l'imatinib [34, 35]. Dans I'étude DADI, Imagawa et al. montrent une rémission sans traitement chez $48 \%$ des patients un an après l'arrêt du dasatinib en seconde ligne de traitement [36]. Dans l'étude STOP-ITK2, au cours de laquelle des patients ont été traités par dasatinib ou nilotinib en première ou seconde ligne de traitement, le taux de RST à 12 et 48 mois après arrêt du traitement, étaient respectivement de 63,33\% (IC $95 \%$ : $51,14 \%-75,53 \%$ ) et $53,57 \%$ (IC $95 \%: 40,49 \%-66,65 \%$ ) [37].

L'absence de rechute franche chez les patients, malgré une maladie résiduelle faiblement détectable, est attribuée, en partie, au contrôle immunologique de la LMC [1], soulignant qu'une éradication totale des cellules souches leucémiques résiduelles n'est pas nécessaire chez tous les patients pour atteindre une RST [1].

Récemment, plusieurs études ont été menées pour identifier les facteurs prédictifs d'un arrêt des ITK réussi, et identifier les patients qui pourraient bénéficier de cette stratégie. Dans l'étude IMMUNOSTIM, dans laquelle ont été suivis 51 patients issus de l'étude STIM, nous avons montré qu'après arrêt de l'imatinib, les patients sans rechute présentaient un nombre plus élevé de cellules NK circulantes, comparativement aux patients qui rechutent. Nous avons par ailleurs montré qu'un taux plus élevé de cellules NK du sous-groupe CD56 dim au moment de l'arrêt du traitement, constitue un facteur pronostique indépendant de survie sans rechute moléculaire [3]. Les études DADI et EUROSKI montrent des résultats similaires: un niveau plus important de cellules NK périphériques est associé à une probabilité plus élevée de rémission sans traitement $[36,38]$. Kimura et al. ont suggéré que l'immunité NK serait plus importante que l'immunité CTL pour obtenir une RST [39]. Dans l'étude IMMUNOSTIM, nous n'avons pas observé d'association entre le nombre de lymphocytes $T \mathrm{CD}^{+} / \mathrm{CD} 4^{+} / \mathrm{CD} 8^{+}$, le ratio CD4/CD8 ou le nombre de lymphocytes Treg chez les patients en rechute, par rapport aux patients qui ne rechutent pas. S'agissant des récepteurs présents à la surface des cellules NK, l'expression de NKp46 et DNAM-1 (DNAX accessory molecule-1, CD226) reste inchangée après l'arrêt du traitement. En revanche, une diminution significative des cellules exprimant NKG2A a été notée chez les patients sans rechute, mais il n'a pas été observé de gain en termes de capacités cytotoxiques ou de sécrétion d'IFN $\gamma$ par les cellules NK du sous-groupe CD56 bright [3]. Hughes et al ont montré que les modifications de la réponse immunitaire obtenues en RMM et RMP après traitement par imatinib, à savoir une plus forte proportion de CD56 $6^{\text {bright }}$ et de $\operatorname{CD} 56^{\text {dim }}$ et une plus faible proportion de MDSC CD33 ${ }^{+} C D 11 b^{-}$ HLADR $^{-}$, de MDSC granulocytaires, de lymphocytes Treg et de lymphocytes $T C D 8^{+} P D 1^{+}$, et un phénotype NK plus mature $\left(\mathrm{CD} 7^{+} \mathrm{CD} 62 \mathrm{~L}^{-}\right)$par rapport au diagnostic, sont maintenues en phase de RST [14].

S'agissant du rôle spécifique des cellules NK dans le succès du traitement par dasatinib, les données restent divergentes. Dans l'étude japonaise D-STOP, on note chez les patients en rechute moléculaire après arrêt du dasatinib, une augmentation de la proportion de cellules NK (avec un taux de rémission sans traitement de $62,9 \%$ à 12 mois) [40]. Au contraire, dans l'étude DADI, un nombre plus élevé de cellules NK ( $C D 3^{-} \mathrm{CD}_{56} 6^{+}$et $\left.\mathrm{CD} 16^{+} \mathrm{CD} 56^{+}\right)$et de cellules NK LGL $\left(\mathrm{CD} 56^{+} \mathrm{CD} 57^{+}\right)$, et un nombre plus faible de lymphocytes $\mathrm{T} \gamma \delta$ et de Treg ( $\left.\mathrm{CD} 25^{+} \mathrm{CD} 127^{\text {low }}\right)$ avant arrêt du dasatinib, sont associés à une meilleure RST (49\% à 6 mois) [36], faisant de ces trois éléments des facteurs de survie sans rechute. Les lymphocytes Treg facilitent l'échappement tumoral. Ils pourraient être un facteur contributif de rechute. Une autre étude ayant porté sur l'arrêt de l'imatinib n'a, quant à elle, rapporté aucune différence en termes de proportion de lymphocytes Treg ou Treg naïfs/mémoire entre les patients en RST et ceux en rechute [41]. Yoshida et al. ont pour leur part suggéré un rôle critique de l'inhibition des lymphocytes Treg par le dasatinib pour l'induction de la différenciation des cellules NK effectrices et l'obtention de la RMP [42]. 


\section{Conclusion et perspectives}

Nos connaissances sur le rôle de l'immunomodulation dans la physiopathologie et le traitement de la LMC s'affinent. La proportion de cellules NK, et particulièrement le sous-type CD $56^{\mathrm{dim}}$ cytotoxique et mature, semble jouer un rôle central. Ces cellules pourraient constituer un important facteur de succès dans l'obtention et le maintien d'une rémission après arrêt d'un traitement par ITK chez les patients en RMP. II n'est cependant pas encore envisageable de fonder la stratégie d'arrêt de traitement par ITK sur cette observation. D'autres stratégies sont à l'étude. L'utilisation d'agents stimulant l'expansion ou la fonction des cellules NK, tels que des anticorps modulant le rôle inhibiteur des récepteurs KIR, est déjà à l'étude dans la leucémie aiguë myéloblastique [43]. Les combinaisons thérapeutiques associant un ITK sont également en cours d'analyse afin d'augmenter les chances d'obtenir une rémission sans traitement: combinaison de l'imatinib avec le lénalinomide (molécule stimulant les cellules NK et les réponses antitumorales) (essai ACTRN12615001169538) ou encore le dasatinib en combinaison avec l'IFN alpha $2 b$ pégylé (essai NCT01872442).

Pour conclure, l'identification d'un (ou de plusieurs) marqueur(s) immunologique(s) robuste(s), permettant de prédire quel patient peut arrêter un traitement par ITK sans risque de rechute, et l'identification des mécanismes immunologiques précis impliqués dans le succès d'une rémission sans traitement restent aujourd'hui une priorité à explorer. $\diamond$

\section{SUMMARY}

NK cells: a major role in the antitumoral immunomodulation in CML Convincing clinical and experimental evidence is converging on the essential role of NK (Natural Killer) cells in the recognition and eradication of tumors. Recent studies emphasized the role of NK cells in the immune control of chronic myeloid leukemia (CML), a malignancy arising from hematopoietic stem cells, and the treatment of which has been revolutionized by the use of tyrosine kinase inhibitors (TKI). Three major findings are emerging: 1) the impairment of the numbers and function of NK cells at diagnosis, 2) the restoration of the NK cell function and numbers during remissions induced with TKI therapies and 3) the potential role of the more mature NK CD56 dim cell population in maintaining relapse-free survival after stopping TKI therapy. Immunological control of CML by NK cells which has been suspected for several decades is thus a new field of investigation for future therapies. $\diamond$

\section{REMERCIEMENTS}

Un soutien financier pour une assistance éditoriale a été fourni par Bristol-Myers Squibb.

\section{LIENS D'INTÉRÊT}

Les auteurs déclarent participer à des réunions et événements scientifiques organisées par les laboratoires Novartis, Bristol-Myers Squibb, Pfizer (Delphine Réa); Bristol-Myers Squibb, Incyte, Novartis, Pfizer (Agnès Guerci-Bresler); Celyad, Innate Pharma, Celgène (Nicolas Dulphy); Bristol-Myers Squibb, Celyad, Innate Pharma (Antoine Toubert). Ali Turhan participe au soutien à la recherche pour Bristol-Myers Squibb et Novartis et au comité consultatif pour les laboratoires Bristol-Myers Squibb, Hybrigenics, Incyte.

\section{RÉFÉRENCES}

1. Hughes $A$, Yong ASM. Immune effector recovery in chronic myeloid leukemia and treatment-free remission. Front Immunol $2017 ; 8: 1-12$.

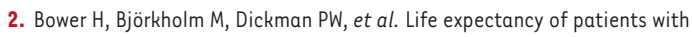
chronic myeloid leukemia approaches the life expectancy of the general population. J Clin Oncol 2016 ; 34 : 2851-58.

3. Rea D, Henry G, Khaznadar Z, Etienne G, et al. Natural killer cell counts are associated with molecular relapse-free survival after imatinib discontinuation in chronic myeloid leukemia : the Immunostim study. Haematol 2017 ; 102 : 1368-77.

4. Yilmaz M, Jabbour $\varepsilon$. Treating early in the disease course: lessons from the CML model. Semin Oncol $2015 ; 42: 876-86$.

5. Rohon P, Porkka K, Mustjoki S. Immunoprofiling of patients with chronic myeloid leukemia at diagnosis and during tyrosine kinase inhibitor therapy. EurJ Haematol $2010 ; 85$ : 387-98.

6. Konjevic G, Vuletic A, Mirjacic Martinovic K. Natural killer cell receptors: alterations and therapeutic targeting in malignancies. Immunol Res 2015; $64: 25-35$.

7. Morvan MG, Lanier LL. NK cells and cancer: you can teach innate cells new tricks. Nat Rev Cancer $2016 ; 16: 7-19$.

8. Pahl J, Cerwenka A. Tricking the balance: NK cells in anti-cancer immunity. Immunobiology 2017 ; 222 : 11-20.

9. Chester C, Fritsch K, Kohrt HE. Natural killer cell immunomodulation: targeting activating, inhibitory, and co-stimulatory receptor signaling for cancer immunotherapy. Front Immunol $2015 ; 6: 601$.

10. Narendra BL, Reddy KE, Shantikumar S, Ramakrishna S. Immune system: a double-edged sword in cancer. Inflamm Res 2013 ; 62 : 823-34.

11. Cheng $M$, Chen $Y$, Xiao W, et al. NK cell-based immunotherapy for malignant diseases. Cell Mol Immunol $2013 ; 10: 230-52$.

12. Mellqvist UH, Hansson M, Brune $M$, et al. Natural killer cell dysfunction and apoptosis induced by chronic myelogenous leukemia cells: role of reactive oxygen species and regulation by histamine. Blood $2000 ; 96: 1961-8$.

13. Chen $\mathrm{Cl}$, Koschmieder $\mathrm{S}$, Kesrtiens $\mathrm{L}$, et al. NK cells are dysfunctional in human chronic myelogenous leukemia before and on imatinib treatment and in BCR-ABL-positive mice. Leukemia $2012 ; 26$ : 465-74.

14. Hughes A, Clarson J, Tang C, et al. CML patients with deep molecular responses to TKI have restored immune effectors and decreased PD- 1 and immune suppressors. Blood 2017 ; 129 : 1166-76.

15. Boissel N, Rea D, Vannary T, Dulphy N. BCR/ABL oncogene directly controls MHC class I chain-related molecule A expression in chronic myelogenous leukemia. J Immunol 2006 ; $176: 5108-16$.

16. Pierson BA, Miller JS. CD56 $6^{+}$bright and $C D 56^{+}$dim natural killer cells in patients with chronic myelogenous leukemia progressively decrease in number, respond less to stimuli that recruit clonogenic natural killer cells, and exhibit decreased proliferation on a per cell basis. Blood $1996 ; 88: 2279-87$.

17. Gabert J, Beillard $\varepsilon$, van der Velden VH, et al. Standardization and quality control studies of real-time quantitative reverse transcriptase polymerase chain reaction of fusion gene transcripts for residual disease detection in leukemia: a Europe against cancer program. Leukemia 2003 ; 17 : 2318-87.

18. Chomel JC, Bonnet ML, Sorel N, et al. Leukemic stem cell persistence in chronic myeloid leukemia patients with sustained undetectable molecular residual disease. Blood $2011 ; 118: 3657-60$.

19. Graham S, Jørgensen HG, Allan $\varepsilon$, et al. Primitive, quiescent, Philadelphiapositive stem cells from patients with chronic myeloid leukemia are insensitive to STI571 in vitro. Blood 2002 ; 99 : 319-25.

20. Chomel JC, Bonnet ML, Sorel N, et al. Evaluation of leukemic stem cell persistence in chronic myeloid leukemia (CML) patients in complete molecular remission induced by first line TKI therapies. Blood 2012; 120 : 3726.

21. Etienne G, Guilhot J, Rea D, et al. Long-term follow-up of the french Stop Imatinib (STIMl) study in patients with chronic myeloid leukemia. J Clin Oncol 2017 ; $35: 298-305$.

22. Salih J, Hilpert J, Placke T, et al. The BCR/ABL-inhibitors imatinib, nilotinib and dasatinib differentially affect NK cell reactivity. Int J Cancer $2010 ; 127$ : 2119-28.

23. Rohon P. Biological therapy and the immune system in patients with chronic myeloid leukemia. Int J Hematol $2012 ; 96$ : 1-9. 


\section{RÉFÉRENCES}

24. Chen $\mathrm{Cl}$, Maecker HT, Lee PP. Development and dynamics of robust T-cell responses to CML under imatinib treatment. Blood $2008 ; 111: 5342-49$.

25. Eissen DN, Michelo CM, Preijers FWMB, et al. Selective expansion of human natural killer cells leads to enhanced alloreactivity. Cell Mol Immunol $2014 ; 11: 160-8$.

26. Kreutzman A, Juvonen V, Kairisto V, et al. Mono-oligoclonal T and NK cells are common in chronic myeloid leukemia patients at diagnosis and expand during dasatinib therapy. Blood $2010 ; 116$ : 772-82.

27. Christiansson L, Söderlund S, Mangsbo S, et al. The tyrosine kinase inhibitors imatinib and dasatinib reduce myeloid suppressor cells and release effector lymphocyte responses. Mol Cancer Ther $2015 ; 14: 1181-91$.

28. El Missiry M, Adnan AS, Al-Samadi A, et al. Assessment of bone marrow lymphocytic status during tyrosine kinase inhibitor therapy and its relation to therapy response in chronic myeloid leukaemia. J Cancer Res Clin Oncol 2016 ; 142 : 1041-50.

29. Mustjoki S, Auvinen K, Kreutzman A, et al. Rapid mobilization of cytotoxic lymphocytes induced by dasatinb therapy. Leukemia $2013 ; 27: 914-24$

30. Piu ZY, Xu W, Li JY. Large granular lymphocytosis during dasatinib therapy. Cancer Biol Ther 2014 ; $15: 247-55$.

31. Iriyama N, Fujisawa S, Yoshida C, et al. Early cytotoxic lymphocyte expansion contributes to a deep molecular response to dasatinib in patients with newly diagnosed chronic myeloid leukemia in the chronic phase: results of the D-first study. Am J Hematol $2015 ; 90: 819-24$.

32. Kim DH, Kamel-Reld S, Chang H, et al. Natural killer or natural killer/T cell lineage large granular lymphocytosis associated with dasatinib therapy for Philadelphia chromosome positive leukemia. Haematol $2009 ; 94: 135-9$

33. Hayashi Y, Nakamae H, Katayama T, et al. Different immunoprofiles in patients with chronic myeloid leukemia treated with imatinib, nilotinib or dasatinib. Leuk Lymphoma 2012 ; 53 : 1084-9.

34. Mahon FX, Réa D, Guilhot J, et al. Discontinuation of imatinib in patients with chronic myeloid leukaemia who have maintained complete molecular remission for at least 2 years: the prospective, multicentre Stop Imatinib (STIM) trial. Lancet Oncol 2010 ; 11 : 1029-35.

35. Ross DM, Brandford S, Seymour JF, et al. Safety and efficacy of imatinib cessation for CML patients with stable undetectable minimal residual disease: results from the TWISTER study. Blood $2013 ; 122: 515-22$.

36. Imagawa J, Tanaka H, Okada M, et al. Discontinuation of dasatinib in patients with chronic myeloid leukemia who have maintained deep molecular response for longer than 1 year (DADI trial): a multicenter phase 2 trial. Lancet Haematol 2015 ; 2 : e528-35.
37. Rea D, Nicolini FE, Tulliez M, et al. Discontinuation of dasatinib or nilotinib in chronic myeloid leukemia : interim analysis of the STOP 2G-TKI study. Blood 2017 ; 129 : 846-54

38. Llander M, Olsson-Strömberg U, Schlums H, et al. Increased proportion of mature NK cells is associated with successful imatinib discontinuation in chronic myeloid leukemia. Leukemia 2017 ; 31 : 1108-16.

39. Kimura $S$. Current status of ABL tyrosine kinase inhibitors stop studies for chronic myeloid leukemia. Stem Cell Investig $2016 ; 3: 36$.

40. Kumagai T, Nakaseko C, Nishiwaki K, et al. Discontinuation of dasatinib after deep molecular response for over 2 years in patients with chronic myelogenous leukemia and the unique profiles of lymphocyte subsets for successful discontinuation: a prospective, multicenter Japanese Trial (D-STOP Trial). Blood 2016; $128: 791$

41. Rea D, Dulphy N, Henry G, et al. Low natural killer (NK) cell counts and functionality are associated with molecular relapse after Imatinib discontinuation in patients (pts) with chronic phase (CP)-chronic myeloid leukemia (CML) with undetectable BCR-ABL transcripts for at least 2 years: Preliminary results from immunostim, On behalf of STIM investigators. Blood $2013 ; 122: 856$.

42. Yoshida C, Iriyama N, Najima Y, et al. Association of peripheral regulatory T cells with achievement of deep molecular response in newly diagnosed chronic phase chronic myeloid leukemia treated with Dasatinib: the final results of D-First study. Blood 2016; $128: 1916$.

43 Vey N, Bourrhis JH, Boissel N, et al. A phase 1 trial of the anti-inhibitory KIR $\mathrm{mAb}$ IPH2101 for AML in complete remission. Blood 2012; 120:4317-23.

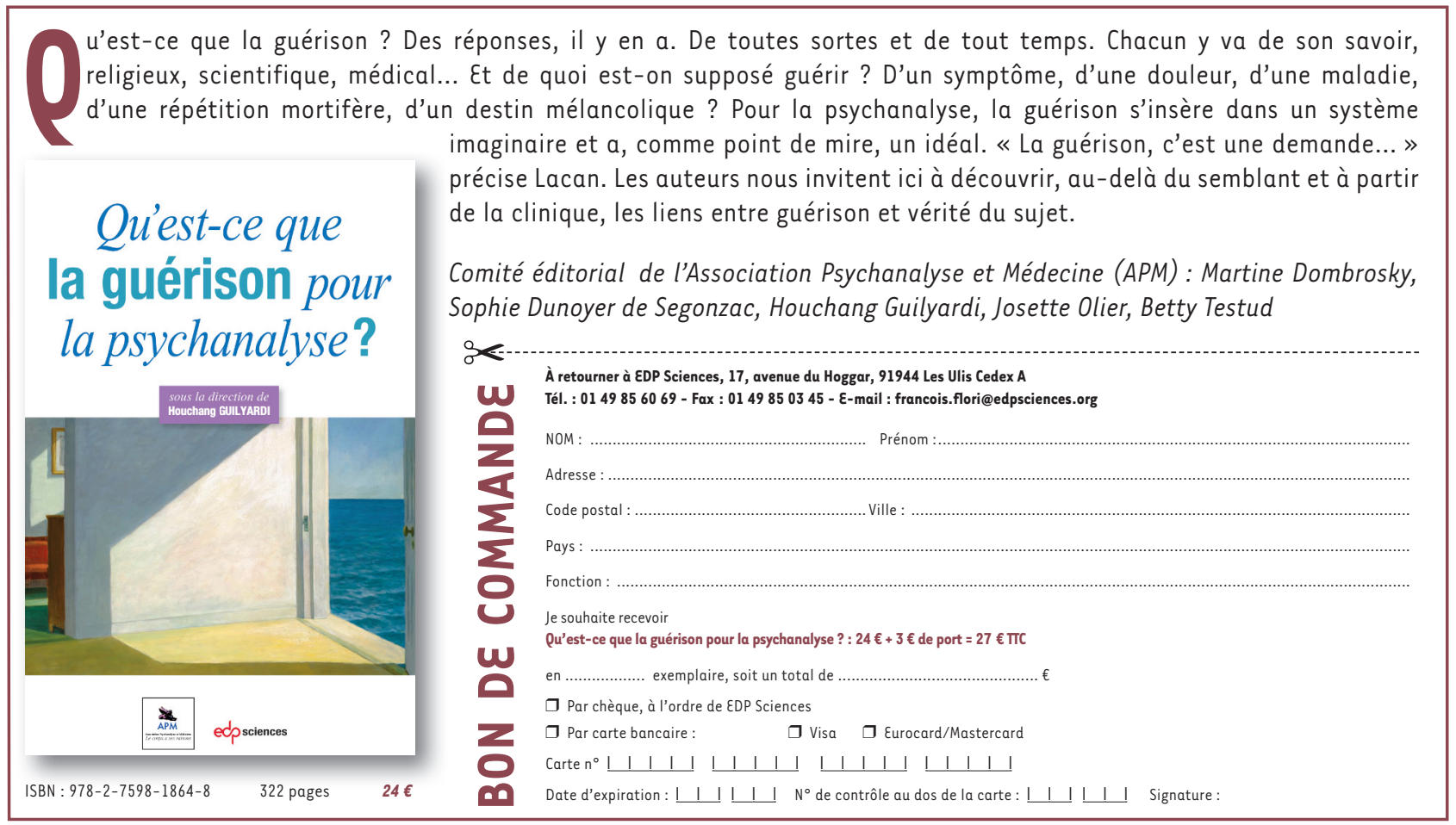

TIRÉS À PART

A. Toubert 